\title{
Efficacy and Safety of Percutaneous Transhepatic Portal Embolization with Dehydrated Ethanol
}

\author{
Ryota Hanaoka $^{1, * \#}$, Tatsuo Banno ${ }^{1, \#}$, Ryoichi Kato ${ }^{2}$, Hokuto Akamatsu $^{1}$ and Hiroshi Toyama ${ }^{1}$ \\ ${ }^{I}$ Department of Radiology, School of Medicine, Fujita Health University, 1-98 Dengakugakubo, Kutsukake, Toyoake, \\ Aichi 470-1192, Japan \\ ${ }^{2}$ Faculty of Radiological Technology, School of Health Sciences, Fujita Health University, 1-98 Dengakugakubo, \\ Kutsukake, Toyoake, Aichi 470-1192, Japan
}

\begin{abstract}
Purpose: The efficacy and safety of percutaneous transhepatic portal embolization (PTPE) with dehydrated ethanol was determined by measuring the liver lobe volume before and after the procedure.

Materials and Methods: A total of 38 patients (25 men, 13 women; mean age: $62.0 \pm 10.8$ years) who underwent PTPE with dehydrated ethanol between April 2005 and March 2011 participated in this study. Dehydrated ethanol containing $17 \%$ lipiodol was injected into the target portal vein branch under balloon occlusion, and the portal vein was subsequently embolized. The liver lobe volume was measured via contrast-enhanced computed tomography, and the percent increase in the unembolized lobe volume was then calculated. In addition, PTPE-related complications were surveyed, and the procedural safety was evaluated.
\end{abstract}

Results: The mean percent increase in the unembolized lobe volume after PTPE was $33.8 \% \pm 20.2 \%$. The procedure could not be completed in one patient because of an insufficient increase in the unembolized lobe volume. No serious postPTPE complications were observed.

Conclusion: These data suggest that PTPE with dehydrated ethanol is a safe and effective method for enlarging the planned residual liver volume before extensive liver resection.

Keywords: Ethanol, hepatectomy, liver cancer, portal vein embolization.

\section{INTRODUCTION}

Makuuchi et al. [1] and Kinoshita et al. [2] introduced percutaneous transhepatic portal vein embolization (PTPE) to the clinic with the intent to induce atrophy in liver areas targeted for resection. At present, preoperative PTPE is used in many hospitals to enlarge the planned residual liver lobes before radical surgery in liver cancer patients. The introduction of PTPE has allowed extensive liver resection even in patients with decreased residual liver function; thus, yielding increased resection rates in patients with liver and bile duct cancer [1-4].

In recent years, technical advances have allowed the development of minimally invasive PTPE procedures. However, the techniques and equipment used in such procedures, including the embolic agents [5-15], vary greatly among facilities [5-15]. The conventional agents include fibrin glue $[2-4,8,12,13]$, gelatin sponges $[14,15]$, dehydrated ethanol (along with the concurrent use of metal

*Address correspondence to this author at the Department of Radiology, Fujita Health University, 1-98, Dengakugakubo, Kutsukake, Toyoake, Aichi 470-1192, Japan; Tel: 81-562-93-9259; Fax: 81-562-95-2253;

E-mail: ryotin@d1.dion.ne.jp

"These authors contributed equally to this work. coils) $[6-9,11,13]$, and monoethanolamine oleate [10]. At our facility, we replaced fibrin glue with dehydrated ethanol as the embolic agent in 2003. Since the first report by Yamakado et al. [11] in 1995, several facilities have adopted PTPE with dehydrated ethanol and have published several reports $[7-9,11,13]$. However, most of the assessments of PTPE efficacy have been based on several post-PTPE measurements performed at individual facilities, percent increases in the unembolized lobe volume, and patient prognosis. Furthermore, only a few reports have investigated the impacts of different embolic agents on the unembolized lobe enlargement ratio $[7-9,11,13]$. The present study compared the liver volume measurements obtained before and after PTPE with dehydrated ethanol to clarify the safety and efficacy of this procedure. In addition, we performed a review of the literature to compare the efficacy of dehydrated ethanol with that of other embolic agents used for PTPE.

\section{MATERIALS AND METHODS}

\section{Patients}

This study was approved by the Institutional Review Board at our institution. 
Table 1. Characteristics of the patients $(n=38)$.

\begin{tabular}{|c|lc|}
\hline Age & 62.0 \pm 10.8 years (range: 36-83 years) \\
\hline \hline \multirow{3}{*}{ Sex } & Male & 25 \\
& Female & 13 \\
\hline \multirow{3}{*}{ Underlying } & Hepatitis B & 3 \\
& Hepatitis C & 3 \\
& Alcoholic hepatitis & 1 \\
& No underlying liver disease & 31 \\
\hline \multirow{5}{*}{ Diseases } & Hepatocellular carcinoma & 10 \\
& Intrahepatic bile duct carcinoma & 7 \\
& Another type of liver cancer & 1 \\
& Metastatic liver cancer & 6 \\
& Hilar cholangiocarcinoma & 9 \\
& Upper biliary tract cancer & 3 \\
& Gallbladder cancer & 2 \\
\hline
\end{tabular}

A total of 38 patients underwent PTPE with dehydrated ethanol prior to liver resection between April 2005 and March 2011. These 38 patients included 25 men and 13 women with a mean age of 62.0 years (range: $36-83$ years). Seven patients had underlying liver diseases, including hepatitis $B(n=3)$, hepatitis $C(n=3)$, and alcoholic hepatitis $(\mathrm{n}=1)$, whereas 31 patients had no underlying liver abnormalities or diseases. The diseases in the latter 31 patients included hepatocellular carcinoma $(\mathrm{n}=10)$, intrahepatic bile duct carcinoma $(n=7)$, another type of liver cancer (undifferentiated embryonal sarcoma; $\mathrm{n}=1$ ), metastatic liver cancer $(n=6)$, hilar cholangiocarcinoma $(n=$ $9)$, upper biliary tract cancer $(\mathrm{n}=3)$, and gallbladder cancer ( $\mathrm{n}=2$; Table 1). Moreover, the inclusion criteria for PTPE were (a) the presence of liver or extrahepatic bile duct cancer, for which a radical cure was expected with extensive liver resection or (b) the presence of gallbladder cancer with a high risk of postoperative liver failure. The exclusion criteria were as follows: a residual hepatic indocyanine green (ICG) plasma clearance (residual liver reserve function index) of $\leq 0.05$ (ICG plasma clearance $\times$ planned residual liver ratio), a standard liver volume (SLV) of <40\% [16] (using the body surface area as a residual liver volume index), and allergies to the iodine contrast medium or ethanol. If patients had been prescribed oral anticoagulants and antiplatelet drugs, these drugs were discontinued 10 days before the PTPE procedure. Patients presenting with obstructive jaundice underwent biliary drainage. Furthermore, PTPE was performed when the serum bilirubin concentration was reduced to $\leq 2 \mathrm{mg} / \mathrm{dL}$.

\section{PTPE Technique}

All patients underwent ultrasound-guided percutaneous transhepatic puncture of the concerned portal vein branch with a percutaneous transhepatic cholangio drainage kit (Cook Japan, Tokyo, Japan). A sheath with a marker (Medikit, Tokyo, Japan) was inserted via guidewire exchange. To further identify a collateral path for the portal vein, contrast imaging of the superior mesenteric and splenic veins was performed using a 4-Fr multipurpose contrast catheter (Terumo Clinical Supply, Gifu, Japan). The diameter of the obstructed portal vein branch was measured by portal venography. A size-2 (lumen) balloon catheter (Terumo Clinical Supply, Gifu, Japan), which was selected according to the portal vein branch diameter, was used to block the portal vein branch targeted for embolization. Adequate blockage of the peripheral side with the balloon was confirmed by contrast imaging as the point at which the flow of contrast medium ceased (Fig. 1). Each patient then received a $20-\mathrm{mg}$ injection of $2 \%$ lidocaine as an analgesic. With the balloon in place, dehydrated ethanol containing $17 \%$ lipiodol was injected into the peripheral side of the balloon until the solution reached the hepatic vein (Fig. 2). The balloon-mediated blockage was continued for $10 \mathrm{~min}$ to optimize efficacy. The balloon blockage was subsequently removed, and thrombi floating around the tip of the balloon catheter were suctioned as much as possible through the ethanol injection site to prevent the outflow of the thrombi into unembolized regions. The balloon catheter was moved forward to the superior mesenteric vein, and portal venography was performed to confirm the embolization. If areas with insufficient embolization were identified, an additional embolization was performed. This procedure was completed by removing the sheath while embolizing the puncture route within the liver parenchyma with a gelatin sponge (Fig. 3).

\section{Volume Measurements}

Contrast computed tomography (CT) images captured before and after PTPE were used to measure the total liver volume as well as the embolized and unembolized lobe volumes and were analyzed on an image-processing workstation (X-LINK or Aquilion-linked workstation; Toshiba Medical Systems, Tochigi, Japan) attached to the CT equipment. All patients underwent multidetector-row CT ( $\geq 16$ rows; Aquilion 16, Aquilion CX, Aquilion Prime, Aquilion One; Toshiba Medical Systems, Tochigi, Japan). Using the hepatic and portal veins as references, the liver region was identified on the workstation console. The regions of interest were manually set on each slice. The areas were then measured and multiplied by the height, to calculate the volumes of the whole liver, embolized lobe, and unembolized lobe (Fig. 4).

\section{Data Analysis}

Prism 5 (GraphPad Software, San Diego, CA, USA) was used for the statistical analysis, and the volume measurements were expressed as means and standard deviations. The volume parameters included the total liver volume, embolized lobe volume, unembolized lobe volume, and ratio of the unembolized lobe volume to the whole liver volume before and after PTPE. The volume measurements obtained before and after PTPE were compared using the Wilcoxon signed-rank test. The patients were divided into two groups, based on the presence or absence of an obstruction or severe stenosis in the primary or secondary portal vein branches after PTPE. The mean percent increases 


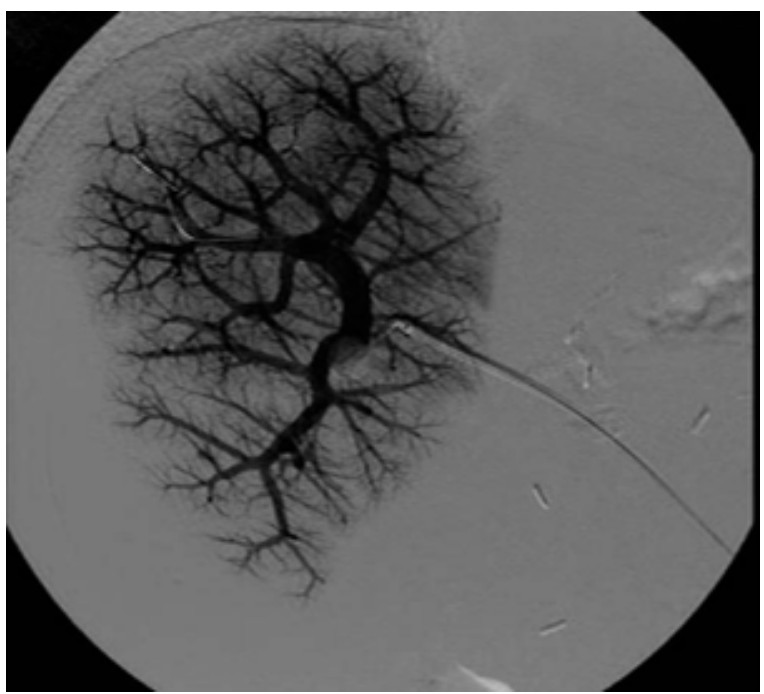

Fig. (1). Right portal vein angiography immediately before injecting absolute ethanol.

A balloon catheter was used to block the portal vein branch intended for embolization. Adequate balloon blockage of the peripheral side was confirmed via contrast imaging when the flow of the contrast medium ceased.

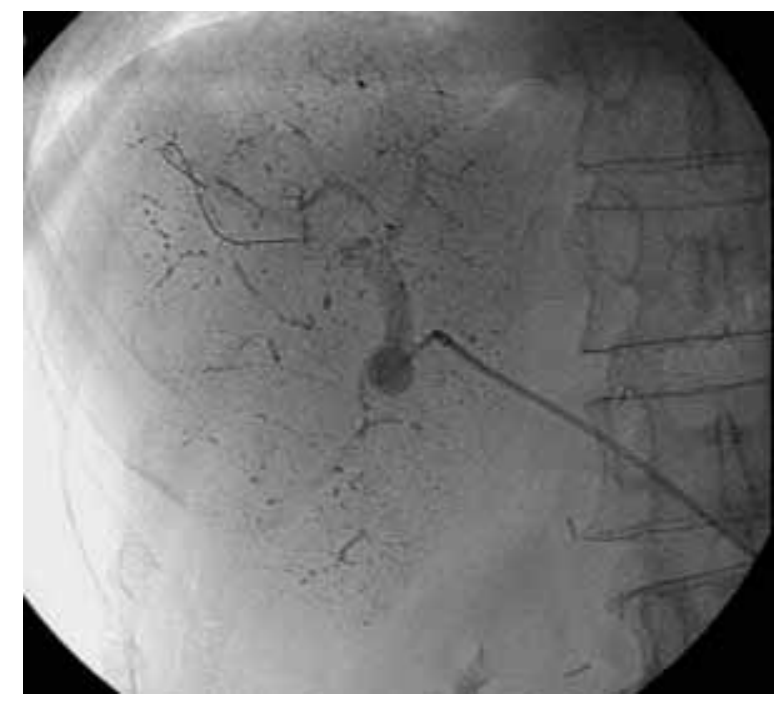

Fig. (2). Fluoroscopic image immediately after injecting absolute ethanol.

With the balloon in place, dehydrated ethanol containing $17 \%$ lipiodol was injected into the peripheral side of the balloon until the solution reached the hepatic vein.

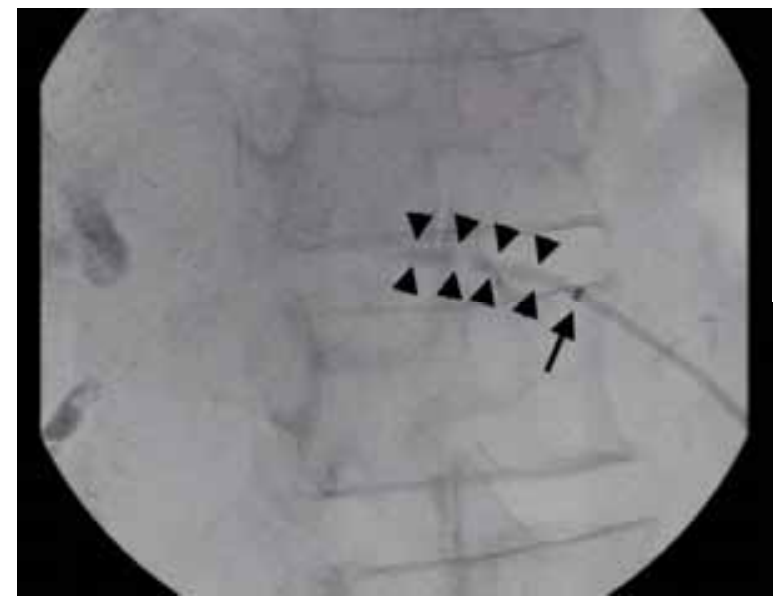

Fig. (3). Embolization of the puncture route.

The procedure was completed by removing the sheath (arrow) while embolizing the puncture route within the liver parenchyma with a gelatin sponge (arrowhead). 


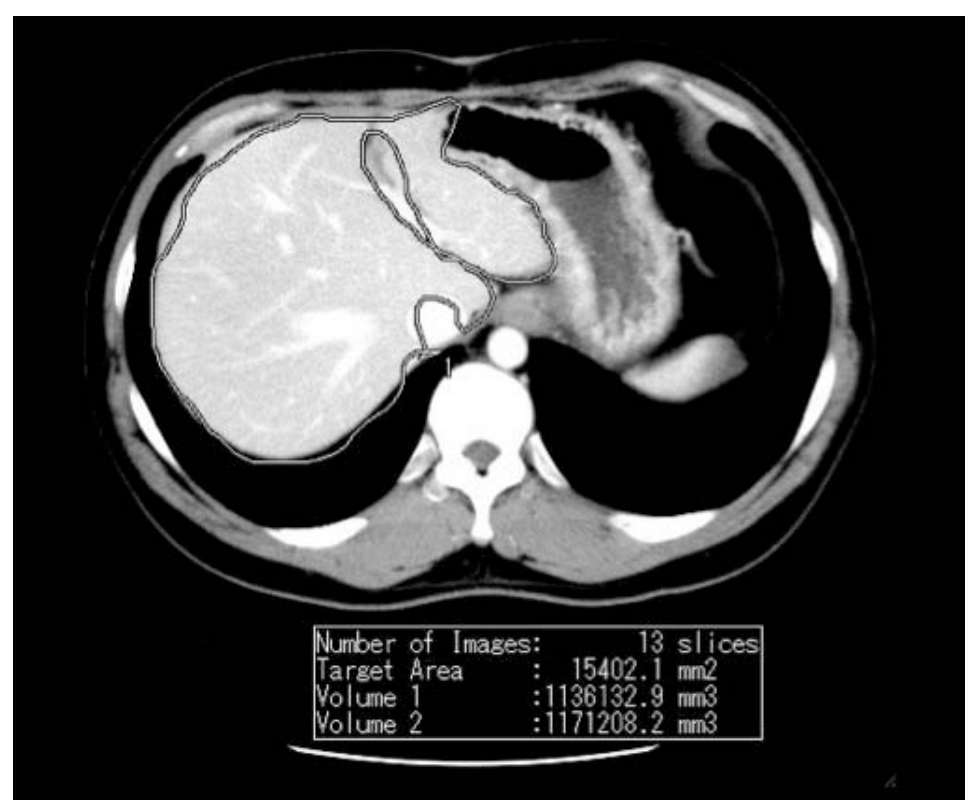

Fig. (4). Liver lobe volume measurements.

The regions of interest (ROI) were set manually on each slice (doubled line). The areas were then measured and multiplied by the height to calculate the volumes of the whole liver, embolized lobe, and unembolized lobe.

Table 2. Change in volume between before and after percutaneous transhepatic portal embolization (PTPE).

\begin{tabular}{|c|c|c|c|}
\hline & Before PTPE & After PTPE & Rate of change \\
\hline \hline Total liver volume & $1144.1 \pm 267.5 \mathrm{~cm}^{3}$ & $1092.7 \pm 243.3 \mathrm{~cm}^{3}$ & $0.97 \pm 0.14$ \\
Unembolized lobe volume & $442.3 \pm 143.1 \mathrm{~cm}^{3}$ & $576.3 \pm 151.8 \mathrm{~cm}^{3}$ & $1.34 \pm 0.20$ \\
Embolized lobe volume & $699.8 \pm 221.5 \mathrm{~cm}^{3}$ & $516.4 \pm 176.4 \mathrm{~cm}^{3}$ & $0.74 \pm 0.17$ \\
Percent liver volume of unembolized lobe & $39 \% \pm 10 \%$ & $53 \% \pm 11 \%$ & \\
\hline
\end{tabular}

in the unembolized lobe volumes of the two groups were compared using the Mann-Whitney $U$ test. For all tests, $p$ values of $<0.05$ were considered statistically significant.

In addition, we monitored the patients for side effects and complications after PTPE with dehydrated ethanol and ascertained their outcomes. Finally, we determined the number of patients who did not subsequently undergo radical surgery and the reasons for their inoperability.

\section{RESULTS}

\section{Characteristics of the Patients}

In 10 patients, occlusion or severe stenosis was observed in the main trunk of the embolized lobe branch. The regions embolized via PTPE were the right portal vein branch in 30 patients, the left portal vein branch and the right anterior branch in four, the left portal vein branch in one, the right anterior branch in one, and the right posterior branch in two. All patients were confirmed to be free of metastasis in the nonembolized liver lobe via Gd-EOB-DTPA contrastenhanced magnetic resonance imaging (MRI) and contrastenhanced CT.

\section{Volume Changes in the Embolized and Unembolized Regions}

Postoperative contrast-enhanced CT revealed that the portal vein branch embolization was successful in all patients. Table $\mathbf{2}$ shows the results of the liver volume measurements. The mean unembolized lobe volume increased from $442.3 \pm 143.1 \mathrm{~cm}^{3}$ before PTPE to $576.3 \pm$ $151.8 \mathrm{~cm}^{3}$ after PTPE $(\mathrm{p}<0.05$; Fig. 5). In contrast, the mean embolized lobe volume decreased from $699.8 \pm 221.5$ $\mathrm{cm}^{3}$ before PTPE to $516.4 \pm 176.4 \mathrm{~cm}^{3}$ after PTPE (p < $0.05)$. Moreover, the mean total liver volume decreased from $1144.1 \pm 267.4 \mathrm{~cm}^{3}$ before PTPE to $1092.7 \pm 243.3 \mathrm{~cm}^{3}$ after PTPE $(\mathrm{p}<0.05)$. The percent liver volume of the unembolized lobe increased from $39.1 \% \pm 9.8 \%$ to $53.5 \% \pm$ $10.7 \%$ ( $p<0.05$; Fig. 6). The percent increase in the unembolized lobe was $33.8 \% \pm 20.2 \%$, and the mean decrease in the embolized lobe was $25.9 \% \pm 16.6 \%$.

\section{Surgery Performance after PTPE}

Of the 38 patients who underwent PTPE, 33 also underwent surgery and none of these patients developed hepatic insufficiency after surgery. The remaining five 


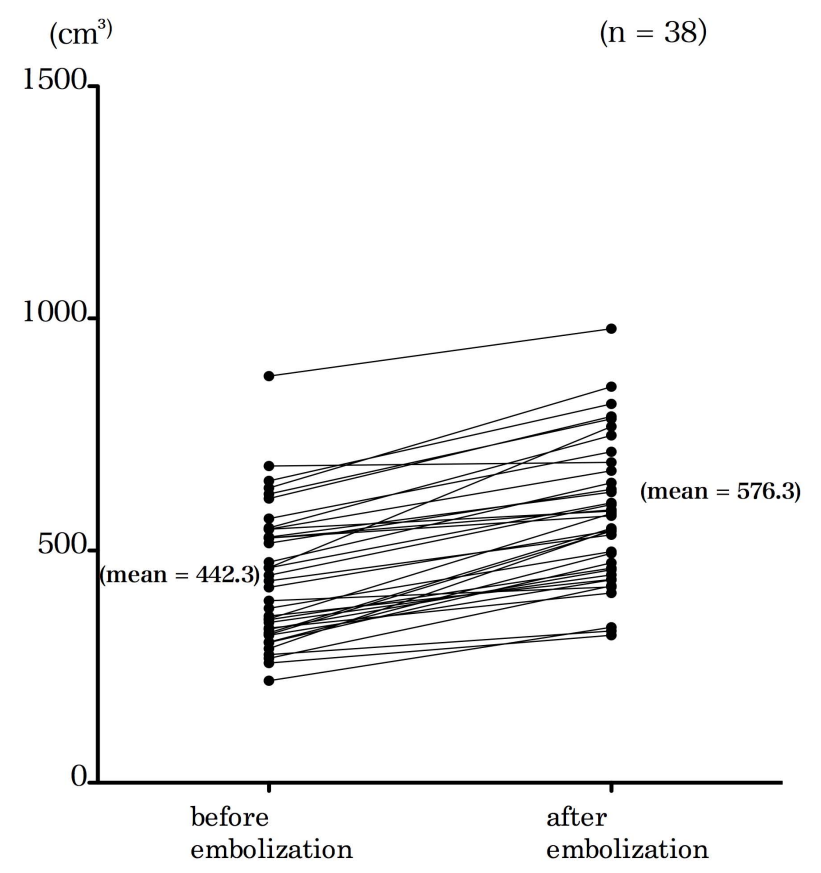

Fig. (5). Volume changes in the unembolized lobes.

The mean unembolized lobe volume significantly increased from $442.3 \pm 143.1 \mathrm{~cm}^{3}$ before percutaneous transhepatic portal embolization (PTPE) to $576.3 \pm 151.8 \mathrm{~cm}^{3}$ after PTPE ( $\left.<<0.05\right)$. The unembolized lobe volumes increased in all cases.

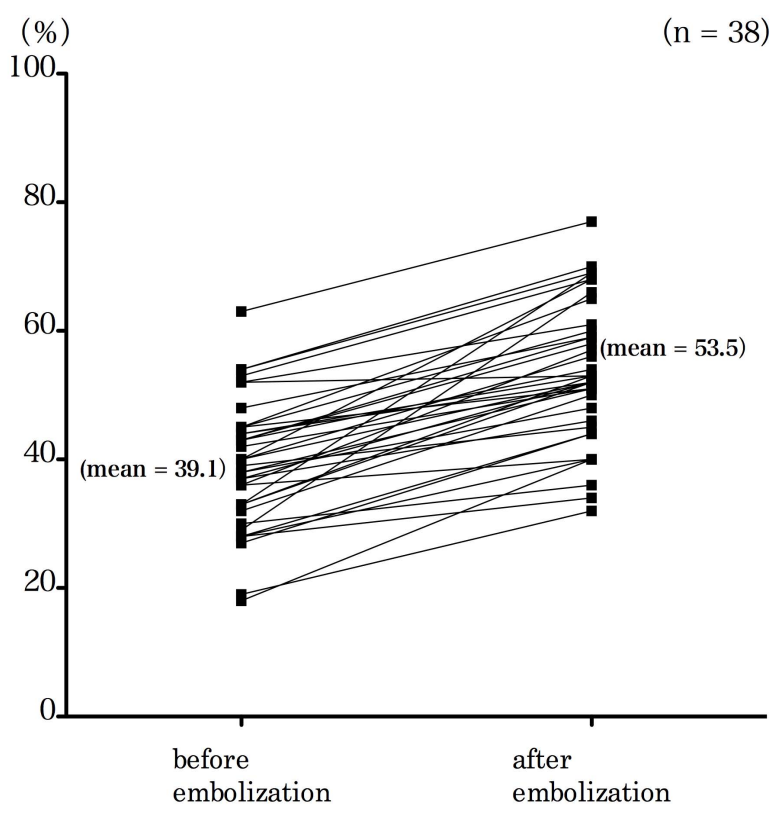

Fig. (6). Changes in the percent liver volume of the unembolized lobe.

The percent liver volume of the unembolized lobe significantly increased from $39.1 \% \pm 9.8 \%$ to $53.5 \% \pm 10.7 \%$ ( $\mathrm{p}<0.05)$.

patients were not approved for surgery either because their conditions progressed while they were waiting for an adequate increase in the unembolized lobe volume $(n=4)$ or because there was an insufficient increase in the unembolized lobe volume $(\mathrm{n}=1)$.

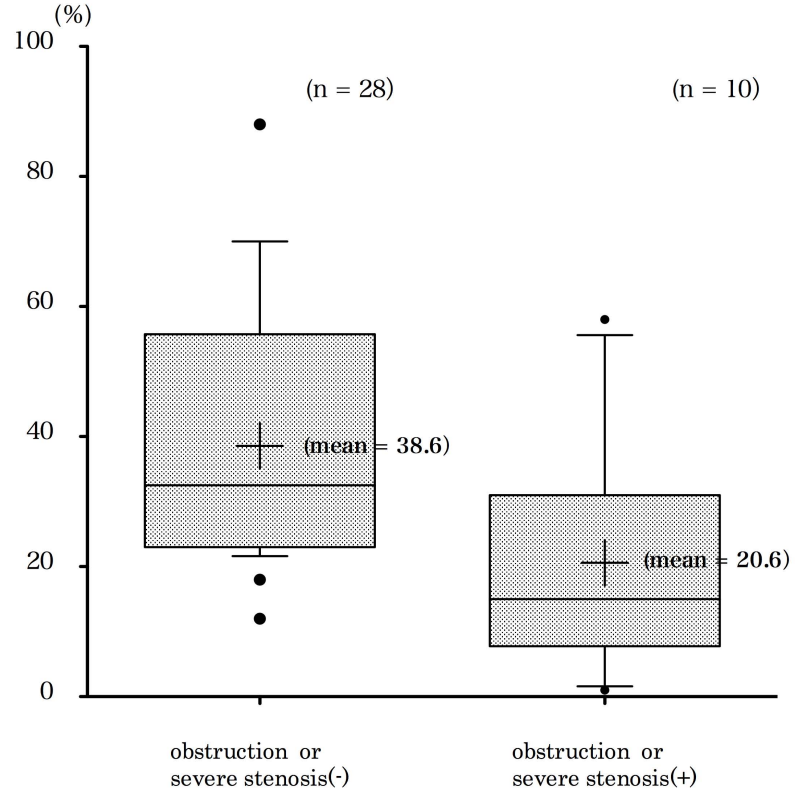

Fig. (7). Impact of postpercutaneous transhepatic portal embolization (PTPE) obstruction or severe stenosis on the unembolized lobe.

Some patients exhibited obstruction or severe stenosis of the portal vein primary or secondary branch after PTPE. In this group, the mean percent increase in the unembolized lobe volume was $20.6 \%$ $\pm 16.4 \%(\mathrm{n}=10) v s .38 .6 \% \pm 19.2 \%(\mathrm{n}=28)$ for patients with no obstruction or stenosis $(\mathrm{p}<0.05)$.

\section{Impact of Post-PTPE Obstruction or Severe Stenosis on the Unembolized Lobe}

Some patients exhibited an obstruction or severe stenosis of the portal vein primary or secondary branch after PTPE. In this group, the mean percent increase in the unembolized lobe volume was $20.6 \% \pm 16.4 \%(\mathrm{n}=10)$, compared with $38.6 \% \pm 19.2 \%(n=28)$ for patients with no obstruction or stenosis ( $\mathrm{p}<0.05$; Fig. 7$)$.

\section{Complications}

During PTPE, all patients complained of abdominal pain during sheath insertion and dehydrated ethanol injection. However, in all cases, this pain subsided after the administration of a local anesthetic and intravenous pentazocine. No patient required emergency surgery after PTPE, and none of the patients developed acute hepatic insufficiency or died. One patient exhibited an outflow of microthrombi into unembolized regions, but we were able to minimize the effects of this condition by using the catheter to push the thrombi into the peripheral portal vein branches. There was an intraoperative case of balloon rupture after dehydrated ethanol injection; however, this was not associated with any adverse events.

\section{DISCUSSION}

The mean percent increase in the unembolized lobe volume after PTPE was $33.8 \% \pm 20.2 \%$. PTPE with dehydrated ethanol was found to be a safe and effective 
method for enlarging the planned residual liver volume prior to extensive liver resection.

Dehydrated ethanol has been reported to cause permanent dehydration of vascular endothelial cells and subsequent coagulation, leading to embolization [11]. Accordingly, dehydrated ethanol has often been used for PTPE or preoperative renal cell carcinoma embolization [17]. Because dehydrated ethanol is a liquid embolic agent, its effects may extend to peripheral bifurcations and the sinusoidal regions [18]. When administered in large doses into the portal vein, dehydrated ethanol is also believed to cause the necrosis of liver cells adjacent to the sinusoidal region [18].

The goal of PTPE is to increase the planned residual liver volume, and thus facilitate extensive liver resection. The PTPE technique with dehydrated ethanol used in the present study yielded considerable increases in the unembolized lobe volume and decreases in the embolized lobe volume in all patients, with an average volume transition of approximately $14 \%$. As the minimal required volume change was approximately $40 \%$ of the $\%$ SLV, the average percent increase in the unembolized lobe volume $(33.8 \% \pm 20.2 \%)$ was sufficient for surgical resection in all but one patient $(97.4 \%)$. The average percent increase in the residual hepatic ICG plasma clearance was $31.7 \% \pm 21.7 \%$, which was equivalent to the average percent increase in the unembolized lobe volume. The exceptional patient had intrahepatic bile duct cancer, from which tumor infiltration had already obstructed the anterior portal vein branch. Therefore, PTPE of the right posterior portal vein branch was performed prior to a planned extensive right hepatectomy; however, the embolized left lobe volume only increased by $1 \%$. Igami et al. [13] reported a mean percent increase in the unembolized lobe volume of $24.0 \%(n=154)$ for PTPE with dehydrated ethanol, which was lower than that achieved by our department. Although the report by Igami et al. did not describe the PTPE technique in detail, the difference may have been because of different dehydrated ethanol injection methods and/or doses.

Previous studies conducted at our facility $(9.9 \% \pm 11.8 \%$; $\mathrm{n}=10$; unpublished data) and another one $(10.2 \% \pm 4.0 \%$; $\mathrm{n}=73$ ) [8] reported lower percent increases in the unembolized lobe volume after PTPE with fibrin glue. These data suggest that dehydrated ethanol induces greater increases in the unembolized lobe volume than fibrin glue, which forms a fibrin clot to obstruct the portal vein lumen [18]. Because fibrin glue has a poor ability to reach the peripheral portal vein branches, it has minimal effects on the vessel walls or liver cells surrounding the portal vein [18]. In contrast, dehydrated ethanol can be used to embolize the peripheral portal vein sites, including the sinusoidal regions, and consequently induce liver cell necrosis. More severe damage to the embolized lobe leads to a greater percent increase of the unembolized lobe. Moreover, a lower embolization success ratio has been reported with fibrin glue than with dehydrated ethanol [8]. Therefore, PTPE with dehydrated ethanol is emerging as a next-generation preoperative procedure for liver cancer patients.

Une et al. [9] reported a mean percent increase in the unembolized lobe volume of $71 \% \pm 25 \%(n=9)$ for PTPE with dehydrated ethanol. This ratio was higher than that achieved at our department. However, most of the patients in the earlier study had hepatocellular carcinoma and had received hepatic arterial injections of an epirubicin-lipiodol suspension 2 weeks before PTPE. Lipiodol, which has a lower embolizing effect when administered alone, embolizes the capillary plexuses around the bile duct and peripheral hepatic arteries [19]. Therefore, even if a major artery is present, the peripheral arterial blood flow will decrease. Lipiodol injections into the hepatic artery before PTPE would accordingly induce a blood flow blockage to the portal arteries and veins in the post-PTPE embolized lobe, leading to more severe liver cell necrosis than that observed after injections of dehydrated ethanol alone. However, if portal vein embolization is performed following hepatic arterial injections of lipiodol, the portal vein blood flow is blocked and the arterial blood flow is decreased in the embolized lobe. This, in turn, greatly decreases the amount of blood flow into the embolized lobe. Therefore, this technique may be associated with a higher risk of extensive liver necrosis and liver failure than that associated with concurrent hepatic arterial injections of lipiodol. If liver failure occurs after PTPE, the waiting period for surgery will be prolonged and the opportunity to perform radical surgery may be lost.

Beppu et al. [10] reported an increase in the percent liver volume of the unembolized lobe from $40 \%$ to $55 \%$ for PTPE with monoethanolamine oleate and gelatin sponges. This ratio was slightly higher than that achieved in our department. Monoethanolamine oleate is a negative ion surfactant that damages vascular endothelial cells and subsequently activates the coagulating system; thus, triggering embolization in a manner similar to that of dehydrated ethanol [10]. However, monoethanolamine oleate is more viscous than dehydrated ethanol, and thus does not leak out of the portal vein even when large amounts are administered. Therefore, monoethanolamine oleate is unlikely to cause necrosis and injury in the surrounding liver cells [10] and is expected to be less efficient than dehydrated ethanol. However, half of the patients in the study by Beppu et al. underwent transarterial chemoembolization before PTPE, and this treatment affected the increase in the unembolized lobe volume.

In the present study, 10 patients developed an obstruction or severe stenosis of the primary or secondary portal vein branches, which was associated with an approximate $50 \%$ decrease in the unembolized lobe volume relative to that in patients without complications. This finding could be attributed to the fact that some embolized lobe reductions occurred prior to PTPE in patients with obstruction and/or severe stenosis of the portal vein in the embolized lobe; therefore, compensatory hypertrophy had already begun to develop in the unembolized lobe.

Dehydrated ethanol injection causes pain and alcoholrelated tissue damage [13]. At our facility, this pain is alleviated via direct injection of the analgesic lidocaine into the portal vein with a catheter immediately before injecting the dehydrated ethanol. Another issue usually encountered when performing PTPE with dehydrated ethanol is the 
corrosive effects of ethanol on the device. Although one patient experienced balloon rupture after ethanol injection, no adverse events were observed during the injection. If balloon rupture occurs during ethanol injection and the maneuver is not immediately stopped, ethanol may flow into the unembolized lobe and cause serious complications. Therefore, caution should be always exercised regarding the possibility of balloon rupture while injecting ethanol.

Another potentially serious complication of PTPE is post-PTPE intraperitoneal hemorrhage, which is caused by intraoperative sheath removal or poor embolization during post-PTPE sheath removal. Because PTPE is performed in the liver parenchyma, where most of the sheath encounters no resistance, sheath position deviations are more likely during this procedure than during routine angiography. Therefore, the sheath is fixed in place with a silk thread or tape to prevent such deviations. Moreover, caution must be exercised because post-PTPE intraperitoneal hemorrhaging may occur if the embolism through the sheath path does not extend far enough into the liver parenchyma. Because the portal vein blood flows into the embolized lobe before embolization, most of the blood flows into the unembolized lobe after embolization, increasing the portal blood pressure in the unembolized lobe. Therefore, a reliable embolization of the puncture path is required if the punctured portal vein branch is included in the unembolized lobe. There are existing reports of puncture path embolization with a metal coil [8, 11]; however, at our facility we perform embolization with a gelatin sponge stick. By filling the sheath with the gelatin sponge, we can correctly position the gelatin sponge stick using the dilator as a pusher.

\section{CONCLUSION}

At our facility, the PTPE technique with dehydrated ethanol yielded a mean 33\% increase in the unembolized lobe volume; thus, allowing extensive liver resection in 37 of the 38 patients. The current literature suggests that dehydrated ethanol is more effective than other embolic agents and does not result in any serious complications. We believe that PTPE with dehydrated ethanol is a safe and effective method for increasing the volume of the planned residual liver prior to extensive liver resection.

\section{CONFLICT OF INTEREST}

The authors confirm that this article content has no conflict of interest.

\section{ACKNOWLEDGEMENTS}

We have not received any funding from any institution for this work.

\section{REFERENCES}

[1] Makuuchi M, Thai BL, Takayasu K, et al. Preoperative portal embolization to increase safety of major hepatectomy for hilar bile duct carcinoma: a preliminary report. Surgery 1990; 107: 521-7.

[2] Kinoshita H, Sakai K, Hirohashi K, Igawa S, Yamasaki O, Kubo S. Preoperative portal vein embolization for hepatocellular carcinoma. World J Surg 1986; 10(5): 803-8.

[3] Nimura Y, Kamiya J, Kondo S, et al. Aggressive preoperative management and extended surgery for hilar cholangiocarcinoma: Nagoya experience. J Hepatobiliary Pancreat Surg 2000; 7: 155-62.

[4] Nagino M, Kamiya J, Uesaka K, et al. Complications of hepatectomy for hilar cholangiocarcinoma. World J Surg 2001; 25: 1277-83.

[5] van Lienden KP, van den Esschert JW, de Graaf W, et al. Portal vein embolization before liver resection: a systematic review. Cardiovasc Intervent Radiol 2013; 36(1): 25-34.

[6] Ogasawara K, Uchino J, Une Y, Fujioka Y. Selective portal vein embolization with absolute ethanol induces hepatic hypertrophy and makes more extensive hepatectomy possible. Hepatology 1996; 23(2) :338-45.

[7] Kubo S, Shiomi S, Tanaka H, et al. Evaluation of the effect of portal vein embolization on liver function by $(99 \mathrm{~m}) \mathrm{Tc}$-galactosyl human serum albumin scintigraphy. J Surg Res 2002; 107(1): 1138.

[8] Ebata T, Yokoyama Y, Igami T, Sugawara G, Takahashi Y, Nagino M. Portal vein embolization before extended hepatectomy for biliary cancer: current technique and review of 494 consecutive embolizations. Dig Surg 2012; 29(1): 23-9.

[9] Une Y, Haneda T, Ogasawara K, et al. Preoperative percutaneous transhepatic portal embolization with absolute ethanol in patients with hepatocellular carcinoma. J Hepatobiliary Pancreat Surg 1997; 4(4): 391-7.

[10] Beppu T, Iwatsuki M, Okabe $\mathrm{H}$, et al. A new approach to percutaneous transhepatic portal embolization using ethanolamine oleate iopamidol. J Gastroenterol 2010; 45: 211-7.

[11] Yamakado K, Takeda K, Nishide Y, et al. Portal vein embolization with steel coils and absolute ethanol: a comparative experimental study with canine liver. Hepatology 1995; 22(6): 1812-8.

[12] Gibo M, Unten S, Yogi A, et al. Percutaneous ipsilateral portal vein embolization using a modified four-lumen balloon catheter with fibrin glue: initial clinical experience. Radiat Med 2007; 25(4): 164-72.

[13] Igami T, Ebata T, Yokoyama Y, Sugawara G, Takahashi Y, Nagino M. Portal vein embolization using absolute ethanol: evaluation of its safety and efficacy. J Hepatobiliary Pancreat Sci 2014; 21(9): 676-81.

[14] Shin JH, Yoon HK, Kwon J, et al. Volumetric analysis of the liver after right portal vein embolization: mid-term follow-up based on embolization score. Clin Radiol 2010; 65(4): 288-96.

[15] Kakizawa H, Toyota N, Arihiro K, et al. Preoperative portal vein embolization with a mixture of gelatin sponge and iodized oil efficacy and safety. Acta Radiol 2006; 47(10): 1022-8.

[16] Urata K, Kawasaki S, Matsunami H, et al. Calculation of child and adult standard liver volume for liver transplantation. Hepatology 1995; $21: 1317-21$

[17] Bakal CW, Cynamon J, Lakritz PS, Sprayregen S. Value of preoperative renal artery embolization in reducing blood transfusion requirements during nephrectomy for renal cell carcinoma. J Vasc Interv Radiol 1993; 4(6): 727-31.

[18] Matsuoka T. Experimental studies of intrahepatic portal vein embolization and embolic materials. Jpn J Radiol 1989; 49: 593606.

[19] Takayasu K, Shima Y, Muramatsu Y, et al. Hepatocellular carcinoma: treatment with intraarterial iodized oil with and without chemotherapeutic agents. Radiology 1987; 162: 345-51.

(C) Hanaoka et al.; Licensee Bentham Open.

This is an open access article licensed under the terms of the Creative Commons Attribution Non-Commercial License (http://creativecommons.org/licenses/bync/3.0/), which permits unrestricted, non-commercial use, distribution and reproduction in any medium, provided the work is properly cited. 